OPEN ACCESS

Edited by:

Li Zuo,

Ohio State University, USA

Reviewed by:

Han-Zhong Feng,

Wayne State University School of

Medicine, USA

Fan Ye,

University of Florida, USA

Feng $\mathrm{He}$,

California State University, Chico, USA

${ }^{*}$ Correspondence:

Iskandar ldris

iskandar.idris@nottingham.ac.uk

Philip J. Atherton

philip.atherton@nottingham.ac.uk

${ }^{\dagger}$ Equal last authors.

Specialty section:

This article was submitted to

Striated Muscle Physiology,

a section of the journal

Frontiers in Physiology

Received: 24 May 2016 Accepted: 08 August 2016 Published: 25 August 2016

Citation:

Rudrappa SS, Wilkinson DJ, Greenhaff PL, Smith K, Idris I and Atherton PJ (2016) Human Skeletal

Muscle Disuse Atrophy: Effects on Muscle Protein Synthesis, Breakdown, and Insulin Resistance-A Qualitative

Review. Front. Physiol. 7:361

doi: 10.3389/fphys.2016.00361

\section{Human Skeletal Muscle Disuse Atrophy: Effects on Muscle Protein Synthesis, Breakdown, and Insulin Resistance-A Qualitative Review}

\author{
Supreeth S. Rudrappa, Daniel J. Wilkinson, Paul L. Greenhaff, Kenneth Smith, \\ Iskandar Idris ${ }^{*+}$ and Philip J. Atherton ${ }^{*+}$ \\ Division of Medical Sciences and Graduate Entry Medicine, School of Medicine, MRC-Arthritis Research UK Centre for \\ Musculoskeletal Ageing Research, Royal Derby Hospital, University of Nottingham, Derby, UK
}

The ever increasing burden of an aging population and pandemic of metabolic syndrome worldwide demands further understanding of the modifiable risk factors in reducing disability and morbidity associated with these conditions. Disuse skeletal muscle atrophy (sometimes referred to as "simple" atrophy) and insulin resistance are "non-pathological" events resulting from sedentary behavior and periods of enforced immobilization e.g., due to fractures or elective orthopedic surgery. Yet, the processes and drivers regulating disuse atrophy and insulin resistance and the associated molecular events remain unclear-especially in humans. The aim of this review is to present current knowledge of relationships between muscle protein turnover, insulin resistance and muscle atrophy during disuse, principally in humans. Immobilization lowers fasted state muscle protein synthesis (MPS) and induces fed-state "anabolic resistance." While a lack of dynamic measurements of muscle protein breakdown (MPB) precludes defining a definitive role for MPB in disuse atrophy, some proteolytic "marker" studies (e.g., MPB genes) suggest a potential early elevation. Immobilization also induces muscle insulin resistance (IR). Moreover, the trajectory of muscle atrophy appears to be accelerated in persistent IR states (e.g., Type II diabetes), suggesting IR may contribute to muscle disuse atrophy under these conditions. Nonetheless, the role of differences in insulin sensitivity across distinct muscle groups and its effects on rates of atrophy remains unclear. Multifaceted time-course studies into the collective role of insulin resistance and muscle protein turnover in the setting of disuse muscle atrophy, in humans, are needed to facilitate the development of appropriate countermeasures and efficacious rehabilitation protocols.

Keywords: skeletal muscle, disuse, immobilization, protein metabolism, diabetes

\section{INTRODUCTION}

Skeletal muscle tissue represents the largest protein/amino acid (AA) reservoir in the human body (Bonaldo and Sandri, 2013). Skeletal muscles are not only crucial for locomotion but also represent the body's largest metabolically active tissue, glucose disposal site, and fuel reservoir for other organs in fasting and pathological conditions (i.e., hepatic supply of amino acids for gluconeogenesis). Loss of muscle mass occurs with many common illnesses (Evans, 2010) including 
cancers (Stephens et al., 2010), renal/heart failure (Gordon et al., 2013), sepsis (Gordon et al., 2013), muscle genetic diseases (Sandri, 2010), and neurodegenerative disorders (Verdijk et al., 2012). Muscle atrophy also occurs in otherwise healthy individuals under situations of reduced neural input-such as during immobilization, e.g., due to leg casting for fractures (Phillips et al., 2009), bed-rest, spinal cord injury (Castro et al., 1999) and during "aging" (i.e., sarcopenia; Evans and Lexell, 1995). The main environmental determinants of muscle mass in adulthood are exogenous essential amino acids (AA) (needing to be acquired through dietary protein intake), Newton's gravity and locomotion (DeFronzo and Tripathy, 2009). Indicative of this, lack of energy intake during starvation (Rennie et al., 2010), inactivity (Wall et al., 2013), spaceflight (Vandenburgh et al., 1999), or limb immobilization (Phillips et al., 2009) all lead to a reduction in muscle cross sectional area (CSA), an associated loss of function, and muscle insulin resistance. Crucially, loss of muscle mass is associated with greater morbidity and mortality (Sasaki et al., 2007), reduced independence, especially in older populations (Leenders et al., 2013) and this is accelerated in type 2 diabetes (Leenders et al., 2013).

\section{Overview of Disuse Atrophy, Countermeasures, and Muscle Metabolism} Disuse atrophy is often referred to as "simple atrophy" in that atrophy is intrinsic to the muscle(s) specifically exposed to disuse; that is, disuse atrophy is not a systemic condition. Countermeasures for disuse atrophy are limited but include forms of mechanical loading (Wilkinson et al., 2008) such as exercise/electrical stimulation (Wall et al., 2012), passive physiotherapy (Fowles et al., 2000) and harnessing the adjunct anabolic effects of protein nutrition (Churchward-Venne et al., 2012b). Ascertaining an understanding of the mechanisms of disuse atrophy-particularly in relation to the regulation of muscle protein synthesis (MPS) and breakdown (MPB)is important for designing countermeasures or rehabilitation protocols (Reggiani, 2015). Furthermore, despite accumulating evidence that physical inactivity plays a causative role in development of non-communicable diseases such as obesity, insulin resistance, type 2 diabetes and dyslipidemia (Atherton et al., 2016), the mechanistic role of muscle insulin resistance (IR) in driving muscle atrophy in the context of "simple disuse" remains unclear (Atherton et al., 2016). This review will focus on identifying different models of human disuse atrophy, the degree of muscle and strength loss and the regulation of muscle protein turnover and muscle IR. Future translational studies to mitigate disuse atrophy will rely upon robust evidence being present in humans of active mechanisms (often of putative mechanisms that have been pre-identified in animals). As such, this review will focus mainly on current evidence from clinical studies.

\section{The Impacts of Experimental Disuse on Muscle Mass and Strength Loss in Humans}

A plethora of clinical studies have investigated the degree of muscle loss in humans exposed to disuse. The most frequent employed models to study disuse atrophy in humans are unilateral limb suspension (ULLS) using a knee brace or cast, and bed rest; other scenarios include spinal cord injury and spaceflight. In terms of muscle mass, the observed rate of decline in muscle size (CSA) for each day of ULLS in knee extensors was $\sim 0.40 \%$ and $\sim 0.36 \%$ for plantar flexors following 42 days of unloading (Hackney and Ploutz-Snyder, 2012). Other studies have demonstrated losses of muscle strength and mass early on in disuse, i.e., 5 days of cast immobilization lead to $\sim 3.5 \%$ reductions in quadriceps CSA and $\sim 9 \%$ in strength (Dirks et al., 2014). This had progressed to $\sim 8 \%$ reductions in CSA and $\sim 23 \%$ reductions in strength by 14 days (Wall et al., 2013). Additionally, Suetta et al. reported $\sim 10 \%$ reductions in myofibre area and $\sim 13 \%$ decreases in strength after just 4 days progressing to $\sim 20 \%$ reductions in myofibre area and strength after 14 days of ULLS (Suetta et al., 2012, 2013). A further study reported decreases in mid-thigh CSA of $11 \%$ following 28 days of bed rest (Brooks et al., 2008). Lastly, a study by Castro et al. showed muscle CSA to be $\sim 45 \%$ less compared to able-bodied controls 6-weeks after complete spinal cord injury (Castro et al., 1999). Adding to the above constellation, Gibson et al. studied men who were immobilized following tibial fracture (thus having 6-weeks of casting) and reported reductions in quadriceps CSA of $\sim 17 \%$. Furthermore, Alkner et al. reported that 90 days bed rest led to $\sim 10$ and $\sim 16 \%$ reductions in quadriceps and triceps surae mass after 29 days, with rates of weekly loss slowing during the last 2 months to roughly half that observed during the first month (Alkner and Tesch, 2004). Finally, muscle CSA decreased by $\sim 5 \%$ (de Boer et al., 2007; Glover et al., 2010) at 14 days and $10 \%$, at 23 days, i.e., $0.5 \%$ day following ULLS (de Boer et al., 2007). Collectively, these studies indicate a varying degree of rates of disuse muscle atrophy, depending on the duration and nature of immobilization but also measurement techniques, i.e., MRI/DXA/ultrasound/ myofibre CSA; however, it appears atrophy occurs more rapidly in first 3-14 days of unloading and eventually reaching a nadir where further loss of muscle occurs at a slower rate despite continued unloading of muscle (Bodine, 2013).

Differences in the rate of muscle atrophy have also been observed according to different muscle and fiber types as well as the mode of immobilization. For example, after prolonged disuse ( $\sim 180$ days of space flight), loss of fiber size and force was reported in the soleus and gastrocnemius muscles with the order of atrophy (greatest-least) being: soleus type I > soleus type II $>$ gastrocnemius type I > gastrocnemius type II (Fitts et al., 2010). Similar effects of disuse on fiber type following 35 days of bed rest was reported in the vastus lateralis (VL) muscle, i.e., the loss of fiber CSA was greater in type 1 than type II fibers (Brocca et al., 2012). Conversely, muscle fiber type specificity has not been observed in other studies (Bamman et al., 1998; Trappe et al., 2008; Hvid et al., 2010) where duration of immobilization was shorter ( $<14$ days). It is notable that, these studies have mainly focused on a single muscle with muscle biopsy taken from a single site in the periphery of the muscle. This is relevant because muscles do not atrophy uniformly across the entire length of a single muscle (Miokovic et al., 2012), with differential atrophy across different muscles being observed following 27-60 days head-down-tilt bed rest. The investigators also reported that the 
posterior calf muscles atrophied faster than the knee extensor muscles (Vastus Lateralis) and ankle flexors (Tibialis anterior). In another study where multiple muscles were examined using MRI over 43 days disuse in the form of ankle immobilization, the greatest rate of muscle loss was observed in soleus and medial gastrocnemius muscle followed by lateral gastrocnemius and tibialis anterior (Psatha et al., 2012). The aetiology driving distinct fiber type, and individual muscle atrophy susceptibility is poorly defined.

\section{Regulation of Skeletal Muscle Mass in Ambulated and Unloaded Human Muscle Ambulated Regulation of MPS and MPB}

Skeletal muscle mass is regulated by the balance between MPS and MPB. Nutrients (i.e., AA) and nutrient derived hormones (i.e., insulin) play a crucial role in regulating the balance between MPS and MPB. In ambulated humans, intake of dietary protein stimulates MPS due to the essential amino acids (EAA) components of proteins (Atherton and Smith, 2012). These anabolic responses are dose-dependent and saturable; at a maximal stimulus, rates of MPS increase $\sim 200-300 \%$ for a period of $\sim 2 \mathrm{~h}$ following $\sim 20 \mathrm{~g}$ protein (Cuthbertson et al., 2005; Atherton and Smith, 2012). In contrast, insulin released following intake of dietary protein and/or $\mathrm{CHO}$, is neither necessary nor sufficient to stimulate MPS (Greenhaff et al., 2008). Reflecting this, the anti-catabolic effects of insulin upon MPB was not recapitulated via AA infusions when insulin concentrations were clamped at $5 \mu \mathrm{U} \cdot \mathrm{ml}^{-1}$ (post absorptive; Greenhaff et al., 2008). Instead, insulin concentrations of just $15 \mathrm{IU} / \mathrm{ml}$ ( $3 \times$ post absorptive) are sufficient to maximally suppress MPB (Wilkes et al., 2009). This anti-catabolic effect of insulin acting on MPB was confirmed in a recent systematic review and meta-analysis of 44 human studies, which concluded insulin did not significantly affect MPS but has a crucial role in reducing MPB (Abdulla et al., 2016). Therefore, while EAA's stimulate MPS, insulin suppresses MPB (and stimulates muscle glucose uptake). On the basis, EAA and insulin are so vital in maintaining muscle metabolic homeostasis, failure of these mechanisms inevitably leads to skeletal muscle atrophy and IR.

\section{Impact of Disuse on MPS and Anabolic Pathways}

Disuse of human skeletal muscle alters muscle metabolism dynamics (Mallinson and Murton, 2013). For instance, early work by Gibson et al. showed that young men exposed to ULLS exhibited $\sim 30 \%$ slower rates of fasted state MPS compared to the contralateral non-immobilized limb (Gibson et al., 1987). Subsequent studies confirmed reductions in MPS; for instance, $\sim 50 \%$ reductions in MPS following 2-weeks of bed rest (Ferrando et al., 1996; Paddon-Jones et al., 2004) and ULLS with braces/casting (Glover et al., 2008). In further agreement with this, Kortebein et al. reported $\sim 30 \%$ reductions in postabsorptive MPS during a $24 \mathrm{~h}$ period in older adults after 10 days of bed rest (Kortebein et al., 2007). Crucially, blunting of MPS in response to muscle unloading is not restricted to fasted periods. Glover et al. reported that ULLS led to $\sim 27 \%$ reductions in postprandial rates of MPS at both low and high doses of AA infusions (Glover et al., 2008). Similarly, Drummond et al. reported that 7 days of bed rest blunted fed state MPS following EAA ingestion (Drummond et al., 2012). Similarly, 14 days of ULLS led to a $\sim 30 \%$ reduction in MPS after ingestion of $20 \mathrm{~g}$ dietary protein (Wall et al., 2013). On this basis, available evidence strongly supports the notion that skeletal muscle atrophy in humans during a period of disuse is driven by blunting of both postabsorptive and postprandial MPS (Rennie et al., 2010; Wall et al., 2013).

The mTOR (mechanistic target of rapamycin) pathway is the major signal transduction network "hub" involved in the regulation of mRNA translation. Cell and rodent based research suggests this system senses important stimuli responsible for the regulation of MPS, i.e., (1) insulin and insulin-like growth factor-1 (IGF-1) through IRS (insulin receptor substrate) and PI3K (Phosphatidylinositol-3) pathways (Wackerhage and Ratkevicius, 2008); (2) AA through leucyl-tRNA/Rag-mTORc1 pathways (Wackerhage and Ratkevicius, 2008), (3) Energy stress through AMPK-eukaryotic elongation factor (AMP activated protein kinase/ eEF2) pathway (Wackerhage and Ratkevicius, 2008), and (4) mechanical stress, e.g., through mechano-sensory pathways (Wackerhage and Ratkevicius, 2008). However, the impact of these factors in the regulation of MPS in human remains unclear. For example, under ambulated conditions, one of the mTOR upstream effector, the class III PI3K hVps34 (human vacuolar protein sorting-34) was shown to be inhibited in amino acid starved state (basal condition) and increased activity of this protein appears to be concomitant with increased S6K1, suggesting it to be key player in mTOR signaling pathway (Dickinson and Rasmussen, 2011). Furthermore, intake of dietary protein typically leads to increase in phosphorylation status of class III PI3K hVps34 (Dickinson and Rasmussen, 2011), mTOR and its downstream substrates regulating mRNA translation, such as $\mathrm{p} 70^{\mathrm{S} 6 \mathrm{~K}}$ (Drummond et al., 2012). However, in response to disuse, blunted phosphorylation of mTOR and p70 ${ }^{\text {S6K }}$ was shown after 2 -weeks of immobilization while, no significant increases in phosphorylation of mTOR and $\mathrm{p} 70^{56 \mathrm{~K}}$ were noted after immobilization (Wall et al., 2013). In contrast, others have reported no decreases in Akt/mTOR signaling despite reductions in MPS (de Boer et al., 2007; Wackerhage and Ratkevicius, 2008). It is worthwhile noting that peak stimulation of signaling proteins (mTOR and $\mathrm{p} 70^{\mathrm{S} 6 \mathrm{~K}}$ ) occurs $1-2 \mathrm{~h}$ after protein intake while many studies have muscle biopsies taken 3-6 h after protein intake when the response would be attenuated or perhaps absent (Glover et al., 2010; West et al., 2011; Churchward-Venne et al., 2012a; Drummond et al., 2012). Further studies are needed with frequent biopsy sampling to fully determine a role for deactivation in mTORC1-related signaling networks (or indeed other putative mechanisms) regulating depressions in post-absorptive and post-prandial MPS.

\section{Impact of Disuse on MPB and Catabolic Pathways}

In contrast to the recognized deficits in MPS during immobilization, the role of MPB in disuse atrophy is less clear. This is partly confounded by the technical and clinical challenges in measuring in vivo rates of MPB (e.g., arterio-venous balance methods to measure rate of appearance i.e., breakdown and pulse chase stable isotope approaches to measure fractional 
breakdown rates; Kumar et al., 2009; Atherton and Smith, 2012; Atherton et al., 2016). In the only study to our knowledge to measure this, Symons et al. demonstrated no increase in MPB in his study of healthy young volunteers exposed to 21 days of microgravity setting using a bed rest model (Symons et al., 2009). Although not directly quantifying MPB, Wall et al. reported that muscle free tracer enrichment over 4-h post prandial period was $>3$ fold higher after immobilization (Wall et al., 2013). Since MPS has consistently been shown to be reduced with immobilization, a likely explanation is that MPB is actually reduced (rather than increased) and hence the less unlabeled phenylalanine efflux was diluting the muscle free labeled tracer (L-(ring- $\left.{ }^{2} \mathrm{H}_{5}\right)$ phenylalanine pool (Wall et al., 2013). Alternatively, an accumulation of free tracer could simply be explained by established "anabolic resistance," i.e., where a failure of AA incorporation into the muscle lead to its accumulation (Glover et al., 2008; Phillips et al., 2009; Rennie, 2009; Wall et al., 2013). Nevertheless, regardless of the driving force behind muscle atrophy (i.e., disuse, aging, cancer, organ failure), blunted postabsorptive and postprandial MPS (Figure 1; anabolic resistance) seem to be the major drivers of disuse atrophy-rather than increases in MPB. Nonetheless, more work is needed across the time-course of unloading to verify this.

In terms of the molecular regulation of MPB, the ubiquitin proteasome system (UPS; Lecker et al., 2006) supplemented by lysosomal and calcium activated calpain (ATP-independent) and caspase dependent cleavage of actinomyosin complexes (Glover et al., 2010) are the major catabolic pathways in muscle. The identification of the "atrogenes" as genes that are uniformly upregulated irrespective of the atrophy stimulus (e.g., denervation, disuse, thermal injury) has received much attention as key drivers of atrophy programming (Bodine et al., 2001a; Jones et al., 2004; Milan et al., 2015). This led to members of the Forkhead Box (Fox) O family (Fox1, 3, and 4) being identified as downstream targets of Akt pathway (Figure 2) and as the main transcription factors regulating MAFbx/atrogin1expression (Sandri et al., 2004). In terms of disuse atrophy, mRNA expression of two E3 ubiquitin ligases was initially found to be crucial in immobilization, unloading and denervation induced muscle atrophy (Bodine et al., 2001b). These genes, MuRF-1 (Muscle Ring Finger-1), and MAFbx/atrogin-1 (Muscle Atrophy F-box), are expressed in skeletal muscle at low levels

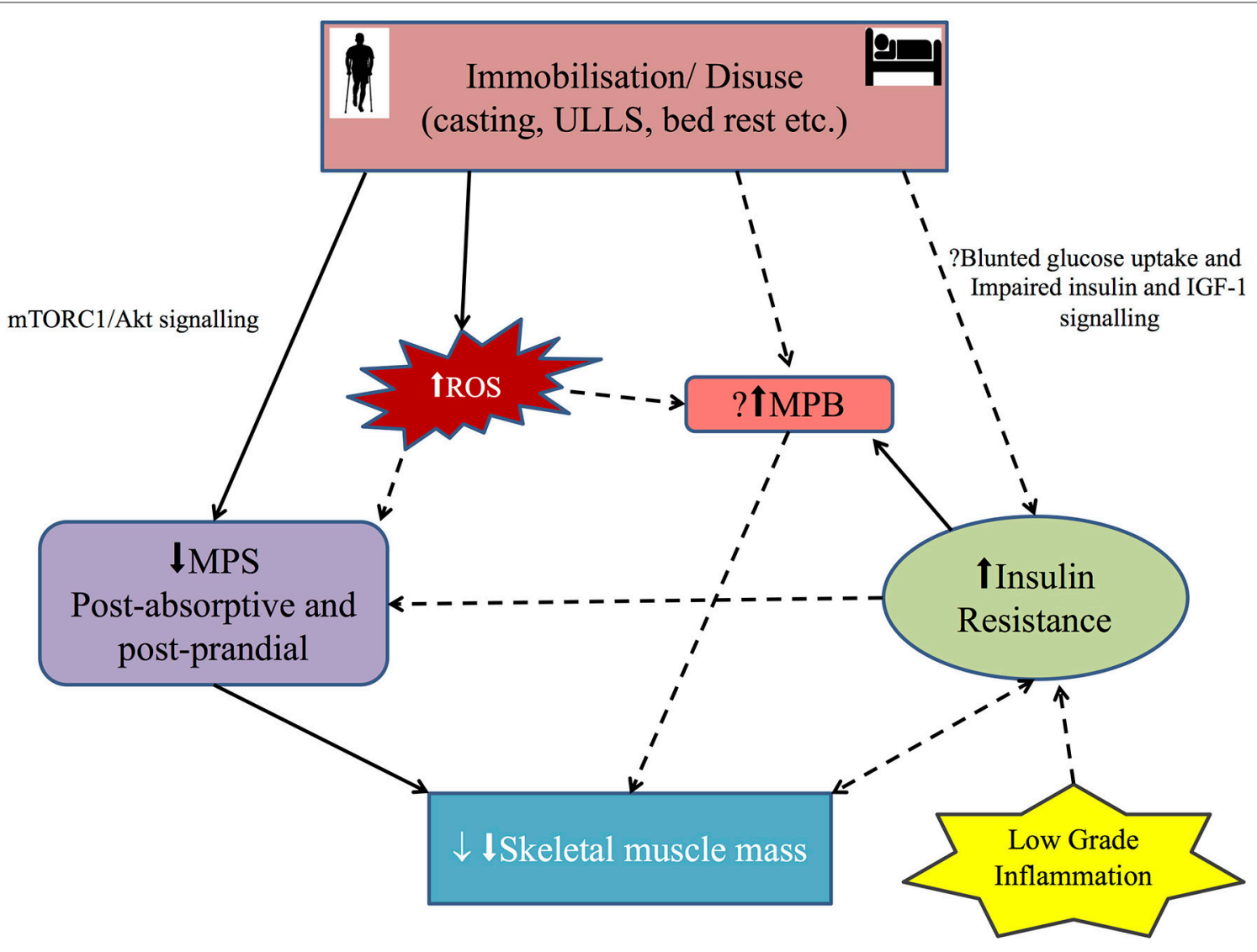

FIGURE 1 | Diagrammatic representation of the main mechanisms involved in disuse skeletal muscle atrophy in humans: Immobilization/disuse reduces both postabsorptive and post prandial muscle protein synthesis (MPS) via the mammalian target of rapamycin (mTORC1) and Akt signaling. The role of MPS, muscle protein breakdown (MPB) and insulin resistance (IR) in simple disuse atrophy remain poorly defined in humans. So the role of insulin resistance and MPB in the setting of disuse atrophy needs further evaluation. Inflammation probably leads to IR. Recently, reactive oxygen species (ROS) has been implicated in development of muscle atrophy in disuse setting, but the mechanism in human remains putative. Solid arrow shows positive association and broken arrow shows putative association. See text for more details. 


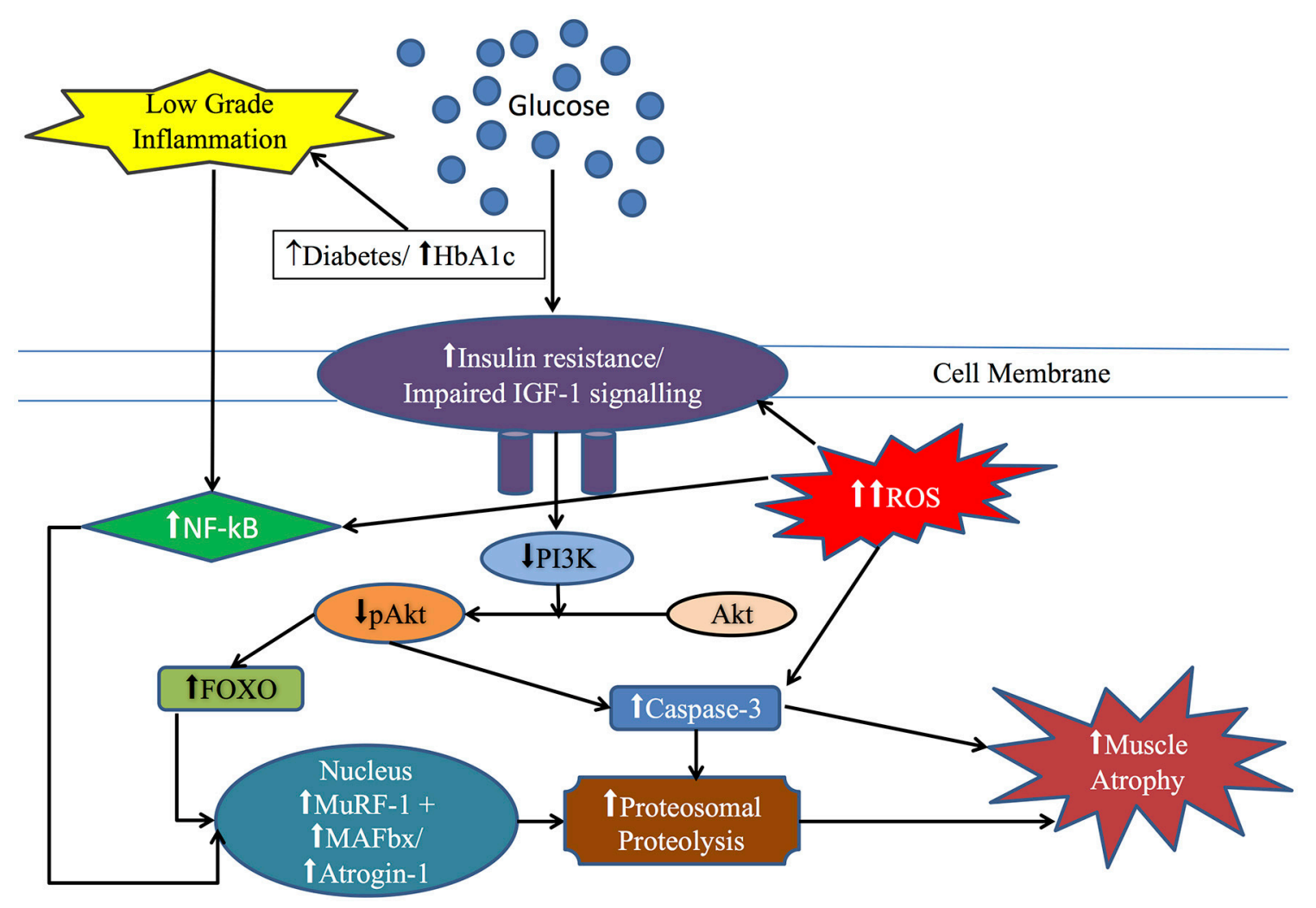

FIGURE 2 | Diagrammatic representation of the overlap between insulin signaling pathway, reactive oxygen species (ROS), inflammatory cytokine such as NF-кB and ubiquitin-proteasome system in insulin resistant (IR) states particularly diabetes: In IR state, PI3K activity is decreased, leading to decreased activity of Akt, which in turn release the inhibition of FOXO and caspase-3 resulting in elevation of muscle ring finger-1 (MuRF-1) and muscle atrophy F-box (MAFbx/atrogin-1) finally leading to increased proteolytic activity. Also, ROS and low grade inflammation via NF- $\mathrm{BB}$ pathway lead to muscle atrophy. See text for more details.

but rapidly induced in response to unloading (Bodine et al., 2001b). In humans, after 5 days (Dirks et al., 2014) and 2 weeks (Jones et al., 2004) of immobilization, MAFbx and MuRF1 mRNA were reported to be elevated. Nonetheless, while their expression is thought to be regulated by transcription factors such as FOXO1, FOXO3a, (Sandri et al., 2004) and NFK $\beta$ (p50 and $\mathrm{Bcl}-3)(\mathrm{Wu}$ et al., 2011), no increase in mRNA expression in FOXO's were noted after 4 or 14 days of immobilization (Suetta et al., 2012). De Boer et al reported the expression of MuRF-1 but not MAFbx mRNA was increased after 10 days of ULLS (Jones et al., 2004; de Boer et al., 2007), while both had decreased by 21 days (de Boer et al., 2007). Furthermore, increases in UPP components (particularly UBE2E) were upregulated $48 \mathrm{~h}$ following instigation of ULLS (Urso et al., 2006). In contrast, a recent study by Brocca et al. found that muscle atrophy following ULLS found no change in mRNA expression of ubiquitin-proteasome and autophagy systems (Brocca et al., 2015). Some work has been done in relation to autophagy (and calpain-signaling) in relation to human disuse (Jones et al., 2004). Autophagy is responsible for removing unfolded, damaged and dysfunctional proteins and organelles via the formation of autophagosomes for degradation by lysosomes (Sandri, 2010). Interestingly, up-regulation of autophagy markers such as Beclin-1 suggested increased autophagosome formation and hence a higher activity of the macro-autophagy by 24 days of bed rest; nonetheless, other autophagy markers such as P62, LC3II/I ratio, and cathepsin-L were not up-regulated (Brocca et al., 2012). While details of the pathways discussed above are outside the scope of this review, the readers are referred to reviews by Bonaldo and Sandri (2013) and Sandri (2010). What is clear however is that without more clinical studies with time-course acquisition, including dynamic measures of MPB in tandem with molecular markers spanning different proteolytic systems, no firm conclusions can be made surrounding the mixed results regarding whether existing molecular data suggest MPB is increased, decreased or unchanged in response to disuse in humans.

In addition to the anabolic and catabolic pathways described above, emerging evidence indicates that disturbed redox signaling may also be an important regulator of MPS and MPB in muscle disuse atrophy (Powers et al., 2012; Zuo and Pannell, 2015). Oxidative injury has been shown to occur in muscle fibers during periods of disuse in locomotor skeletal muscles (Min et al., 2011) and in non-load bearing muscle such as the diaphragm during prolonged mechanical ventilation (Kavazis et al., 2009). After 35 days of bed rest, vastus lateralis muscle showed $\sim 18 \%$ 
muscle fiber atrophy and increased protein carbonylation (Dalla Libera et al., 2009). Furthermore, an inverse linear relationship was observed between normalized levels of protein oxidation and muscle fiber CSA (Dalla Libera et al., 2009). An analysis of gene expression showed up-regulation of pathways involved in the oxidative stress response (increase in mRNA for stress response gene heme oxygenase-1) following $48 \mathrm{~h}$ of unilateral lower leg suspension (ULLS; Reich et al., 2010). Conversely, in a limb immobilization human model with $\sim 5.7 \%$ muscle and $11.8 \%$ muscle fiber loss after 14 days of immobilization, no increase in lipid peroxidation and protein oxidation in vastus lateralis was observed (Glover et al., 2010). Although information on oxidative stress and potential mechanisms explaining proteolysis in disuse human muscle is still sparse (Pellegrino et al., 2011), these findings support the extensive evidence available from animal studies that oxidative stress inhibits MPS (Powers et al., 2011a) and increases muscle MPB (via increased gene expression of key proteins involved in the proteolytic pathways such as autophagy, calpains and proteosomes; activation of both calpain and caspase3 and possibly by modification of myofibrillar proteins which enhances their susceptibility to proteolytic processing; Powers et al., 2011a). Interaction between ROS and insulin signaling pathway has also been described, i.e., ROS may regulate Insulin growth factor-1 (IGF-1) signaling either positively or negatively depending on the amount of ROS produced (Papaconstantinou, 2010). Low levels of endogenous ROS due to their reversible oxidative inhibition of protein tyrosine phosphatases induces phosphorylation of tyrosine residue on the insulin receptor and its substrates triggering IGF-1 signaling (Bashan et al., 2009). In contrast, the IGF-1 signaling pathway is inhibited by higher levels of ROS and recent evidence suggests ROS down regulates the IGF-1 cascade and induces insulin resistance (Bashan et al., 2009; Figure 2). For detailed discussion of the signaling pathways linking ROS and muscle atrophy, the interested reader is referred to recent reviews on oxidative stress and disuse muscle atrophy (Pellegrino et al., 2011; Powers et al., 2011b, 2014; Zuo and Pannell, 2015).

\section{Impact of Disuse on Muscle IR and Links to Muscle Mass in Persistent IR States}

Insulin-mediated glucose uptake is also blunted with muscle disuse (Mikines et al., 1991; Biensø et al., 2012); that is, unloaded muscle becomes IR. This IR can be observed at a whole-body level following bed-rest, but is most apparent at the muscle level across the physiological range of insulin concentrations under clamp conditions (Mikines et al., 1991). Recently, a 1 week bedrest study in young males by Dirks et al. revealed reduced muscle mass ( $\sim 1.4 \mathrm{~kg}$ lean tissue and $\sim 3 \%$ quadriceps CSA) and wholebody insulin sensitivity ( 29\%)(Dirks et al., 2016). Thus, disuse lowers MPS, induces anabolic resistance to nutrients and impairs insulin-mediated muscle glucose uptake-even in healthy adults (Fink et al., 1983).

The role of IR in driving muscle atrophy however is poorly defined. Evidence from large cross-sectional and longitudinal studies reports accelerated loss of muscle mass in individuals with persistent IR (i.e., people with Type 2 Diabetes), perhaps pointing to mechanistic links. For instance, declines in muscle mass were inversely related to duration of diabetes or $\mathrm{HbA1c}$ (Park et al., 2007, 2009; Kalyani et al., 2013) and attenuated with insulin sensitizers (Kuo et al., 2009). Human muscle tissue accounts for $80 \%$ of glucose uptake after food ingestion and insulin resistance (HOMA-IR) is associated with reduced quadriceps muscle strength (Kalyani et al., 2013; Leenders et al., 2013), power (Kalyani et al., 2013) and muscle mass (Leenders et al., 2013) in humans. Approximately a 50\% more rapid decline in knee extensor strength has been observed in older patients with type 2 diabetes compared with patients without diabetes over a 3 year period, suggesting that decreased muscle strength may be accelerated in type 2 diabetes (Park et al., 2007). In a further study, Volpato et al. reported differences in walking speed, muscle strength, power and muscle quality between individuals with and without diabetes were independent of co-existing peripheral motor neuropathy or peripheral vascular disease, suggesting a direct effect of diabetes per se on muscle performance (Volpato et al., 2012). These findings are important because in catabolic conditions such as diabetes, atrophy in combination with reduced activity decrease quality of life and increase mortality (Zinna and Yarasheski, 2003). Yet despite clear evidence linking accelerated muscle loss in diabetes compared to non-diabetes, studies investigating the direct effect of immobilization on muscle protein turnover in patients with diabetes compared to those without diabetes are scant. Furthermore, clear distinction between Type 1 and Type 2 diabetes needs to be made when investigating patients with diabetes. This is because Type 1 diabetes is a condition with severe depletion of energy stores and reduced mitochondrial function resulting in accelerated muscle protein loss (Hebert and Nair, 2010), which can be reversed by insulin replacement (Workeneh and Bajaj, 2013). In contrast, muscle loss, whilst accelerated in type 2 diabetes, is unaffected by insulin treatment (Workeneh and Bajaj, 2013), possibly due to IR. Hence, skeletal muscle mass loss whilst common, appears to occur less predictably and to varying degree in Type 2 diabetes compared with Type 1 diabetes (Workeneh and Bajaj, 2013). Collectively, these data are consistent with the notion that diabetes causes muscle mass loss possibly due to mechanisms driving muscle IR, however there is lack of data regarding the effects of immobilization or disuse on muscle mass in individuals with diabetes.

The mechanistic regulation of muscle IR in driving muscle atrophy in the setting of "simple disuse" remains vague (Atherton et al., 2016). Early human studies by Shulman et al. showed that, under steady state plasma concentration of both glucose and insulin mimicking postprandial conditions, the mean rate of muscle glycogen synthesis accounted for most of the whole body glucose uptake and virtually all of non-oxidative glucose metabolism in both healthy and diabetic subjects (Shulman, 2000), with defects in muscle glycogen synthesis playing a major role in causing insulin resistance in type 2 diabetes (Shulman, 2000). This may be explained by defects in the insulin receptor substrate (IRS)-1/phosphatidylinositol (PI) 3kinase pathway, leading to reduced glucose uptake and utilization in insulin target tissues (Draznin, 2006). Free fatty acids induce muscle IR by inhibiting glucose transport/phosphorylation and 
reductions in both the rate of muscle glycogen synthesis and glucose oxidation (Roden et al., 1996). Additionally, many other mechanisms have been postulated to explain free fatty acid-induced muscle IR, including the Randle cycle, oxidative stress, inflammation and mitochondrial dysfunction (Martins et al., 2012). Full details regarding above mechanisms escape the scope of this article and readers are referred to a review by Martins et al. (2012). With regard to disuse induced muscle atrophy, following (7 days) bed-rest healthy volunteers showed reduced glucose infusion rate and leg glucose extraction (after bed rest) along with reduced muscle GLUT4, hexokinase II, protein kinase $\mathrm{B} / \mathrm{Akt} 1$, and Akt2 protein levels, and a tendency for reduced 3-hydroxyacyl-CoA dehydrogenase activity (Biensø et al., 2012). Further in the same study, the ability of insulin to phosphorylate Akt and activate glycogen synthase was reduced post bed-rest (Biensø et al., 2012); but whether this observation is causative or a consequence of immobilization is not clear. However, a substantial decline in glucose uptake within 24 $\mathrm{h}$ of immobilization would argue against a causative effect (Atherton et al., 2016). Recently, Vigelso et al. showed an inverse association between the increase in muscle pyruvate dehydrogenase complex (PDC) activation and leg lactate release during contraction after 2 weeks unilateral lower limb immobilization, suggesting PDC as a potential key regulator of immobilization induced muscle IR (Vigelsø et al., 2016). Overall the above data suggests that muscle disuse results in development of whole body and muscle specific IR, fuelling the argument that lack of muscle contraction per se may be the main physiological driver for this dysregulation, however a mechanistic explanation for this still remains unclear (Atherton et al., 2016).

\section{CONCLUSIONS}

Disuse muscle atrophy causes many undesirable complications. There seems to be complex interplay of numerous mechanisms contributing to the aetiology of disuse muscle atrophy. During muscle disuse, both post-absorptive and post-prandial MPS is suppressed, with little evidence to support there being an increase in "bulk" protein MPB. Moreover, animal models show increased (2.5 times) rate of muscle protein turnover and are also very sensitive to disuse, while exhibiting marked fiber-type-dependant

\section{REFERENCES}

Abdulla, H., Smith, K., Atherton, P. J., and Idris, I. (2016). Role of insulin in the regulation of human skeletal muscle protein synthesis and breakdown: a systematic review and meta-analysis. Diabetologia 59, 44-55. doi: 10.1007/ s00125-015-3751-0

Alkner, B. A., and Tesch, P. A. (2004). Knee extensor and plantar flexor muscle size and function following 90 days of bed rest with or without resistance exercise. Eur. J. Appl. Physiol. 93, 294-305. doi: 10.1007/s00421-0041172-8

Atherton, P. J., Paul, L. G., Stuart, M. P., Sue, C. B., Christopher, M. A., and Charles, H. L. (2016). Control of skeletal muscle atrophy in response to disuse: clinical/preclinical contentions and fallacies of evidence. Am. J. Physiol. Endocrinol. Metab. doi: 10.1152/ajpendo.00257.2016. [Epub ahead of print]. differences in rates of muscle protein turnover (type I fibers being twice as great as type II fibers) when compared to humans. Due to these inherent species-specific differences, pre-clinical findings cannot easily be reconciled with nor extrapolated to humans. So, further research quantifying MPS and MPB and their temporal relationship during disuse in humans is warranted. There is strong evidence that type 2 diabetes accelerates muscle loss, possibly due to mechanisms innate to diabetes. Crucially, muscle IR secondary to disuse appears to drive the procession of disuse muscle atrophy independent of other mechanisms known to cause muscle IR. Nonetheless, the mechanistic role of muscle IR driving this atrophic response is poorly defined. Because, common proteolytic mechanisms may exist across "simple muscle atrophy" and other catabolic conditions (e.g., type 2 diabetes, inflammation, cachexia etc.), these two process can rarely be seen as being mutually exclusive (Atherton et al., 2016). Further, many questions remain unanswered especially the molecular regulation of MPS and MPB and muscle insulin resistance. This whole area of research has potential implications for the wider clinical community as similar metabolic processes occur during cancer cachexia, metabolic syndromes including type 2 diabetes, aging (i.e., sarcopenia), sepsis and many neurodegenerative disorders. Henceforth, further translational research is necessary before this knowledge can be effectively applied in developing targeted strategies to prevent this in the setting of disuse muscle atrophy.

\section{AUTHOR CONTRIBUTIONS}

All authors listed, have made substantial, direct and intellectual contribution to the work, and approved it for publication.

\section{ACKNOWLEDGMENTS}

The first author is a doctoral research student funded through the University of Nottingham within the MRC-ARUK Centre for Musculoskeletal Ageing Research. The MRC-ARUK Centre for Musculoskeletal Ageing Research was funded through grants from the Medical Research Council [grant number MR/K00414X/1] and Arthritis Research UK [grant number 19891] awarded to the Universities of Nottingham and Birmingham.
Atherton, P. J., and Smith, K. (2012). Muscle protein synthesis in response to nutrition and exercise. J. Physiol. 590, 1049-1057. doi: 10.1113/jphysiol.2011. 225003

Bamman, M. M., Clarke, M. S., Feeback, D. L., Talmadge, R. J., Stevens, B. R., Lieberman, S. A., et al. (1998). Impact of resistance exercise during bed rest on skeletal muscle sarcopenia and myosin isoform distribution. J. Appl. Physiol. (1985) 84, 157-163.

Bashan, N., Kovsan, J., Kachko, I., Ovadia, H., and Rudich, A. (2009). Positive and Negative regulation of insulin signaling by reactive oxygen and nitrogen species. Physiol. Rev. 89, 27-71. doi: 10.1152/physrev.00014.2008

Biensø, R. S., Ringholm, S., Kiilerich, K., Aachmann-Andersen, N. J., KroghMadsen, R., Guerra, B., et al. (2012). GLUT4 and Glycogen Synthase are key players in bed rest-induced insulin resistance. Diabetes 61, 1090-1099. doi: $10.2337 / \mathrm{db} 11-0884$ 
Bodine, S. C., Latres, E., Baumhueter, S., Lai, V. K., Nunez, L., Clarke, B. A., et al. (2001a). Identification of ubiquitin ligases required for skeletal muscle atrophy. Science 294, 1704-1708. doi: 10.1126/science.1065874

Bodine, S. C., Stitt, T. N., Gonzalez, M., Kline, W. O., Stover, G. L., Bauerlein, R., et al. (2001b). Akt/mTOR pathway is a crucial regulator of skeletal muscle hypertrophy and can prevent muscle atrophy in vivo. Nat. Cell Biol. 3, 1014-1019. doi: 10.1038/ncb1101-1014

Bodine, S. C. (2013). Hibernation: the search for treatments to prevent disuse-induced skeletal muscle atrophy. Exp. Neurol. 248, 129-135. doi: 10.1016/j.expneurol.2013.06.003

Bonaldo, P., and Sandri, M. (2013). Cellular and molecular mechanisms of muscle atrophy. Dis. Model. Mech. 6, 25-39. doi: 10.1242/dmm.010389

Brocca, L., Cannavino, J., Coletto, L., Biolo, G., Sandri, M., Bottinelli, R., et al. (2012). The time course of the adaptations of human muscle proteome to bed rest and the underlying mechanisms. J. Physiol. 590, 5211-5230. doi: 10.1113/jphysiol.2012.240267

Brocca, L., Longa, E., Cannavino, J., Seynnes, O., de Vito, G., McPhee, J., et al. (2015). Human skeletal muscle fibre contractile properties and proteomic profile: adaptations to 3 weeks unilateral lower limb suspension and active recovery. J. Physiol. 24, 5361-5385. doi: 10.1113/jp271188

Brooks, N., Cloutier, G. J., Cadena, S. M., Layne, J. E., Nelsen, C. A., Freed, A. M., et al. (2008). Resistance training and timed essential amino acids protect against the loss of muscle mass and strength during 28 days of bed rest and energy deficit. J. Appl. Physiol. (1985) 105, 241-248. doi: 10.1152/japplphysiol. 01346.2007

Castro, M. J., Apple, D. F. Jr., Staron, R. S., Campos, G. E., and Dudley, G. A. (1999). Influence of complete spinal cord injury on skeletal muscle within 6 mo of injury. J. Appl. Physiol. 86, 350-358.

Churchward-Venne, T. A., Burd, N. A., Mitchell, C. J., West, D. W., Philp, A., Marcotte, G. R., et al. (2012a). Supplementation of a suboptimal protein dose with leucine or essential amino acids: effects on myofibrillar protein synthesis at rest and following resistance exercise in men. J. Physiol. 590, 2751-2765. doi: 10.1113/jphysiol.2012.228833

Churchward-Venne, T. A., Burd, N. A., and Phillips, S. M. (2012b). Nutritional regulation of muscle protein synthesis with resistance exercise: strategies to enhance anabolism. Nutr. Metab. 9:40. doi: 10.1186/1743-7075-9-40

Cuthbertson, D., Smith, K., Babraj, J., Leese, G., Waddell, T., Atherton, P., et al. (2005). Anabolic signaling deficits underlie amino acid resistance of wasting, aging muscle. FASEB J. 19, 422-424. doi: 10.1096/fj.04-2640fje

Dalla Libera, L., Ravara, B., Gobbo, V., Tarricone, E., Vitadello, M., Biolo, G., et al. (2009). A transient antioxidant stress response accompanies the onset of disuse atrophy in human skeletal muscle. J. Appl. Physiol. (1985) 107, 549-557. doi: 10.1152/japplphysiol.00280.2009

de Boer, M. D., Selby, A., Atherton, P., Smith, K., Seynnes, O. R., Maganaris, C. N., et al. (2007). The temporal responses of protein synthesis, gene expression and cell signalling in human quadriceps muscle and patellar tendon to disuse. J. Physiol. 585, 241-251. doi: 10.1113/jphysiol.2007. 142828

DeFronzo, R. A., and Tripathy, D. (2009). Skeletal muscle insulin resistance is the primary defect in type 2 diabetes. Diabetes Care 32(Suppl. 2), 157-163. doi: $10.2337 / \mathrm{dc} 09-\mathrm{S} 302$

Dickinson, J. M., and Rasmussen, B. B. (2011). Essential amino acid sensing, signaling, and transport in the regulation of human muscle protein metabolism. Curr. Opin. Clin. Nutr. Metab. Care 14, 83-88. doi: 10.1097/MCO.0b013e3 $283406 f 3 \mathrm{e}$

Dirks, M. L., Wall, B. T., Snijders, T., Ottenbros, C. L., Verdijk, L. B., and van Loon, L. J. (2014). Neuromuscular electrical stimulation prevents muscle disuse atrophy during leg immobilization in humans. Acta Physiol. 210, 628-641. doi: 10.1111/apha. 12200

Dirks, M. L., Wall, B. T., van de Valk, B., Holloway, T. M., Holloway, G. P., Chabowski, A., et al. (2016). One week of bed rest leads to substantial muscle atrophy and induces whole-body insulin resistance in the absence of skeletal muscle lipid accumulation. Diabetes. doi: 10.2337/db15-1661. [Epub ahead of print].

Draznin, B. (2006). Molecular mechanisms of insulin resistance: serine phosphorylation of insulin receptor substrate-1 and increased expression of p85alpha: the two sides of a coin. Diabetes 55, 2392-2397. doi: 10.2337/db060391
Drummond, M. J., Dickinson, J. M., Fry, C. S., Walker, D. K., Gundermann, D. M., Reidy, P. T., et al. (2012). Bed rest impairs skeletal muscle amino acid transporter expression, mtorcl signaling, and protein synthesis in response to essential amino acids in older adults. Am. J. Physiol. Endocrinol. Metab. 302, E1113-E1122. doi: 10.1152/ajpendo.00603.2011

Evans, W. J. (2010). Skeletal muscle loss: cachexia, sarcopenia, and inactivity. Am. J. Clin. Nutr. 91, 1123S-1127S. doi: 10.3945/ajcn.2010.28608A

Evans, W. J., and Lexell, J. (1995). Human aging, muscle mass, and fiber type composition. J. Gerontol. Series A Biol. Sci. Med. Sci. 50, 11-16. doi: 10.1093/gerona/50A.Special_Issue.11

Ferrando, A. A., Lane, H. W., Stuart, C. A., Davis-Street, J., and Wolfe, R. R. (1996). Prolonged bed rest decreases skeletal muscle and whole body protein synthesis. Am. J. Physiol. Endocrinol. Metab. 270, E627-E633.

Fink, R. I., Kolterman, O. G., Griffin, J., and Olefsky, J. M. (1983). Mechanisms of insulin resistance in ageing. J. Clin. Invest. 71, 1523-1535.

Fitts, R. H., Trappe, S. W., Costill, D. L., Gallagher, P. M., Creer, A. C., Colloton, P. A., et al. (2010). Prolonged space flight-induced alterations in the structure and function of human skeletal muscle fibres. J. Physiol. 588, 3567-3592. doi: 10.1113/jphysiol.2010.188508

Fowles, J. R., MacDougall, J. D., Tarnopolsky, M. A., Sale, D. G., Roy, B. D., and Yarasheski, K. E. (2000). The effects of acute passive stretch on muscle protein synthesis in humans. Can. J. Appl. Physiol. 25, 165-180. doi: 10.1139/h00-012

Gibson, J. N., Halliday, D., Morrison, W. L., Stoward, P. J., Hornsby, G. A., Watt, P. W., et al. (1987). Decrease in human quadriceps muscle protein turnover consequent upon leg immobilization. Clin. Sci. 72, 503-509. doi: $10.1042 / \mathrm{cs} 0720503$

Glover, E. I., Phillips, S. M., Oates, B. R., Tang, J. E., Tarnopolsky, M. A., Selby, A., et al. (2008). Immobilization induces anabolic resistance in human myofibrillar protein synthesis with low and high dose amino acid infusion. J. Physiol. 586, 6049-6061. doi: 10.1113/jphysiol.2008.160333

Glover, E. I., Yasuda, N., Tarnopolsky, M. A., Abadi, A., and Phillips, S. M. (2010). Little change in markers of protein breakdown and oxidative stress in humans in immobilization-induced skeletal muscle atrophy. Appl. Physiol. Nutr. Metab. 35, 125-133. doi: 10.1139/H09-137

Gordon, B. S., Kelleher, A. R., and Kimball, S. R. (2013). Regulation of muscle protein synthesis and the effects of catabolic states. Int. J. Biochem. Cell Biol. 45, 2147-2157. doi: 10.1016/j.biocel.2013.05.039

Greenhaff, P. L., Karagounis, L. G., Peirce, N., Simpson, E. J., Hazell, M., Layfield, R., et al. (2008). Disassociation between the effects of amino acids and insulin on signaling, ubiquitin ligases, and protein turnover in human muscle. Am. J. Physiol. Endocrinol. Metab. 295, E595-E604. doi: 10.1152/ajpendo. 90411.2008

Hackney, K. J., and Ploutz-Snyder, L. L. (2012). Unilateral lower limb suspension: integrative physiological knowledge from the Past 20 Years (1991-2011). Eur. J. Appl. Physiol. 112, 9-22. doi: 10.1007/s00421-011-1971-7

Hebert, S. L., and Nair, K. S. (2010). Protein and energy metabolism in type 1 diabetes. Clin. Nutr. 29, 1-11. doi: 10.1016/j.clnu.2009.09.001

Hvid, L., Aagaard, P., Justesen, L., Bayer, M. L., Andersen, J. L., Ørtenblad, N., et al. (2010). Effects of aging on muscle mechanical function and muscle fiber morphology during short-term immobilization and subsequent retraining. J. Appl. Physiol. 109, 1628-1634. doi: 10.1152/japplphysiol. 00637.2010

Jones, S. W., Hill, R. J., Krasney, P. A., O’Conner, B., Peirce, N., and Greenhaff, P. L. (2004). Disuse atrophy and exercise rehabilitation in humans profoundly affects the expression of genes associated with the regulation of skeletal muscle mass. FASEB J. 18, 1025-1027. doi: 10.1096/fj.03-1228fje

Kalyani, R. R., Tra, Y., Yeh, H. C., Egan, J. M., Ferrucci, L., and Brancati, F. L. (2013). Quadriceps strength, quadriceps power, and gait speed in older, u.s. adults with diabetes mellitus: results from the national health and nutrition examination survey, 1999-2002. J. Am. Geriatr. Soc. 61, 769-775. doi: 10.1111 /jgs. 12204

Kavazis, A. N., Talbert, E. E., Smuder, A. J., Hudson, M. B., Nelson, W. B., and Powers, S. K. (2009). Mechanical ventilation induces diaphragmatic mitochondrial dysfunction and increased oxidant production. Free Radic. Biol. Med. 46, 842-850. doi: 10.1016/j.freeradbiomed.2009.01.002

Kortebein, P., Ferrando, A., Lombeida, J., Wolfe, R., and Evans, W. J. (2007). Effect of 10 days of bed rest on skeletal muscle in healthy older adults. JAMA 297, 1772-1774. doi: 10.1001/jama.297.16.1772-b 
Kumar, V., Atherton, P., Smith, K., and Rennie, M. J. (2009). Human muscle protein synthesis and breakdown during and after exercise. J. Appl. Physiol. 106, 2026-2039. doi: 10.1152/japplphysiol.91481.2008

Kuo, C. K., Lin, L. Y., Yu, Y. H., Wu, K. H., and Kuo, H. K. (2009). Inverse association between insulin resistance and gait speed in nondiabetic older men: results from the U.S. National Health and Nutrition Examination Survey (NHANES) 1999-2002. BMC Geriatrics 9:49. doi: 10.1186/1471-2318-9-49

Lecker, S. H., Goldberg, A. L., and Mitch, W. E. (2006). Protein degradation by the ubiquitin-proteasome pathway in normal and disease states. J. Am. Soc. Nephrol. 17, 1807-1819. doi: 10.1681/ASN.2006010083

Leenders, M., Verdijk, L. B., van der Hoeven, L., Adam, J. J., van Kranenburg, J., Nilwik, R., et al. (2013). Patients with type 2 diabetes show a greater decline in muscle mass, muscle strength, and functional capacity with aging. J. Am. Med. Dir. Assoc. 14, 585-592. doi: 10.1016/j.jamda.2013.02.006

Mallinson, J. E., and Murton, A. J. (2013). Mechanisms responsible for disuse muscle atrophy: potential role of protein provision and exercise as countermeasures. Nutrition 29, 22-28. doi: 10.1016/j.nut.2012.04.012

Martins, A. R., Nachbar, R. T., Gorjao, R., Vinolo, M. A., Festuccia, W. T., Lambertucci, R. H., et al. (2012). Mechanisms underlying skeletal muscle insulin resistance induced by fatty acids: importance of the mitochondrial function. Lipids Health Dis. 11:30. doi: 10.1186/1476-511X-11-30

Mikines, K. J., Richter, E. A., Dela, F., and Galbo, H. (1991). Seven days of bed rest decrease insulin action on glucose uptake in leg and whole body. J. Appl. Physiol. 70, 1245-1254.

Milan, G., Romanello, V., Pescatore, F., Armani, A., Paik, J. H., Frasson, L., et al. (2015). Regulation of autophagy and the ubiquitin-proteasome system by the foxo transcriptional network during muscle atrophy. Nat. Commun. 6:6670. doi: 10.1038/ncomms7670

Min, K., Smuder, A. J., Kwon, O. S., Kavazis, A. N., Szeto, H. H., and Powers, S. K. (2011). Mitochondrial-Targeted antioxidants protect skeletal muscle against immobilization-induced muscle atrophy. J. Appl. Physiol. (1985) 111, 1459-1466. doi: 10.1152/japplphysiol.00591.2011

Miokovic, T., Armbrecht, G., Felsenberg, D., and Belavý, D. L. (2012). Heterogeneous atrophy occurs within individual lower limb muscles during 60 days of bed rest. J. Appl. Physiol. 113, 1545-1559. doi: 10.1152/japplphysiol. 00611.2012

Paddon-Jones, D., Sheffield-Moore, M., Zhang, X. J., Volpi, E., Wolf, S. E., Aarsland, A., et al. (2004). Amino acid ingestion improves muscle protein synthesis in the young and elderly. Am. J. Physiol. Endocrinol. Metab. 286, E321-E328. doi: 10.1152/ajpendo.00368.2003

Papaconstantinou, J. (2010). Insulin/IGF-1 and ROS signaling pathway cross-talk in aging and longevity determination. Mol. Cell Endocrinol. 299, 89-100. doi: 10.1016/j.mce.2008.11.025

Park, S. W., Goodpaster, B. H., Lee, J. S., Kuller, L. H., Boudreau, R., de Rekeneire, N., et al. (2009). Excessive loss of skeletal muscle mass in older adults with type 2 diabetes. Diabetes Care 32, 1993-1997. doi: 10.2337/dc09-0264

Park, S. W., Goodpaster, B. H., Strotmeyer, E. S., Kuller, L. H., Broudeau, R., Kammerer, C., et al. (2007). Accelerated loss of skeletal muscle strength in older adults with type 2 diabetes: the health, aging, and body composition study. Diabetes Care 30, 1507-1512. doi: 10.2337/dc06-2537

Pellegrino, M. A., Desaphy, J. F., Brocca, L., Pierno, S., Camerino, D. C., and Bottinelli, R. (2011). Redox homeostasis, oxidative stress and disuse muscle atrophy. J. Physiol. 589, 2147-2160. doi: 10.1113/jphysiol.2010.203232

Phillips, S. M., Glover, E. I., and Rennie, M. J. (2009). Alterations of protein turnover underlying disuse atrophy in human skeletal muscle. J. Appl. Physiol. (1985) 107, 645-654. doi: 10.1152/japplphysiol.00452.2009

Powers, S. K., Smuder, A. J., and Criswell, D. S. (2011a). Mechanistic links between oxidative stress and disuse muscle atrophy. Antioxid. Redox Signal. 15, 2519-2528. doi: 10.1089/ars.2011.3973

Powers, S. K., Smuder, A. J., and Judge, A. R. (2012). Oxidative stress and disuse muscle atrophy: cause or consequence? Curr. Opin. Clin. Nutr. Metab. Care 15, 240-245. doi: 10.1097/MCO.0b013e328352b4c2

Powers, S. K., Talbert, E. E., and Adhihetty, P. J. (2011b). Reactive oxygen and nitrogen species as intracellular signals in skeletal muscle. J. Physiol. 589, 2129-2138. doi: 10.1113/jphysiol.2010.201327

Powers, S. K., Kavazi, A. N., and McClung, J. M. (2014). Oxidative stress and disuse muscle atrophy. J. Appl. Physiol. 102, 2389-2397. doi: 10.1152/japplphysiol. 01202.2006
Psatha, M., Wu, Z., Gammie, F. M., Ratkevicius, A., Wackerhage, H., Lee, J. H., et al. (2012). A longitudinal mri study of muscle atrophy during lower leg immobilization following ankle fracture. J. Magn. Reson. Imaging 35, 686-695. doi: 10.1002/jmri.22864

Reggiani, C. (2015). Not all disuse protocols are equal: new insight into the signalling pathways to muscle atrophy. J. Physiol. 593, 5227-5228. doi: 10.1113/JP271613

Reich, K. A., Chen, Y. W., Thompson, P. D., Hoffman, E. P., and Clarkson, P. M. (2010). Forty-Eight hours of unloading and $24 \mathrm{~h}$ of reloading lead to changes in global gene expression patterns related to ubiquitination and oxidative stress in humans. J. Appl. Physiol. 109, 1404-1415. doi: 10.1152/japplphysiol. 00444.2010

Rennie, M. J. (2009). Anabolic resistance: the effects of aging, sexual dimorphism, and immobilization on human muscle protein turnover. Appl. Physiol. Nutr. Metab. 34, 377-381. doi: 10.1139/H.09-012

Rennie, M. J., Selby, A., Atherton, P., Smith, K., Kumar, V., Glover, E. L., et al. (2010). Facts, noise and wishful thinking: muscle protein turnover in aging and human disuse atrophy. Scand. J. Med. Sci. Sports 20, 5-9. doi: 10.1111/j.16000838.2009.00967.x

Roden, M., Price, T. B., Perseghin, G., Petersen, K. F., Rothman, D. L., Cline, G. W. et al. (1996). Mechanism of free fatty acid-induced insulin resistance in humans. J. Clin. Invest. 97, 2859-65. doi: 10.1172/JCI118742

Sandri, M. (2010). Autophagy in skeletal muscle. FEBS Lett. 584, 1411-1416. doi: 10.1016/j.febslet.2010.01.056

Sandri, M., Sandri, C., Gilbert, A., Skurk, C., Calabria, E., Picard, A., et al. (2004). Foxo transcription factors induce the atrophy- related ubiquitin ligase atrogin-1 and cause skeletal muscle atrophy. Cell 117, 1-2. doi: 10.1016/j.jsbmb.2011.07.002.Identification

Sasaki, H., Kasagi, F., Yamada, M., and Fujita, S. (2007). Grip strength predicts cause-specific mortality in middle-aged and elderly persons. Am. J. Med. 120, 337-342. doi: 10.1016/j.amjmed.2006.04.018

Shulman, G. I. (2000). Cellular mechanisms of insulin resistance. J. Clin. Invest. 106, 171-176. doi: 10.1172/JCI10583

Stephens, N. A., Gallagher, I. J., Rooyackers, O., Skipworth, R. J., Tan, B. H., Marstrand, T., et al. (2010). Using transcriptomics to identify and validate novel biomarkers of human skeletal muscle cancer cachexia. Genome Med. 2:122. doi: $10.1186 /$ gm 122

Suetta, C., Frandsen, U., Jensen, L., Jensen, M. M., Jespersen, J. G., Hvid, L. G., et al. (2012). Aging affects the transcriptional regulation of human skeletal muscle disuse atrophy. PLoS ONE 7:e51238. doi: 10.1371/journal.pone. 0051238

Suetta, C., Frandsen, U., Mackey, A. L., Jensen, L., Hvid, L. G., Bayer, M. L., et al. (2013). Ageing is associated with diminished muscle re-growth and myogenic precursor cell expansion early after immobility-induced atrophy in human skeletal muscle. J. Physiol. 591, 3789-3804. doi: 10.1113/jphysiol.2013. 257121

Symons, T. B., Sheffield-Moore, M., Chinkes, D. L., Ferrando, A. A., and PaddonJones, D. (2009). Artificial gravity maintains skeletal muscle protein synthesis during 21 days of simulated microgravity. J. Appl. Physiol. (1985) 107, 34-38. doi: 10.1152/japplphysiol.91137.2008

Trappe, S., Creer, A., Minchev, K., Slivka, D., Louis, E., Luden, N., et al. (2008). Human soleus single muscle fiber function with exercise or nutrition countermeasures during 60 days of bed rest. Am. J. Physiol. 294, R939-R947. doi: 10.1152/ajpregu.00761.2007

Urso, M. L., Scrimgeour, A. G., Chen, Y. W., Thompson, P. D., and Clarkson, P. M. (2006). Analysis of human skeletal muscle after $48 \mathrm{~h}$ immobilization reveals alterations in mrna and protein for extracellular matrix components. J. Appl. Physiol. 101, 1136-1148. doi: 10.1152/japplphysiol.00180.2006

Vandenburgh, H., Chromiak, J., Shansky, J., Del Tatto, M., and Lemaire, J. (1999). Space travel directly induces skeletal muscle atrophy. FASEB J. 13, 1031-1038.

Verdijk, L. B., Dirks, M. L., Snijders, T., Prompers, J. J., Beelen, M., Jonkers, R. A., et al. (2012). Reduced satellite cell numbers with spinal cord injury and aging in humans. Med. Sci. Sports Exerc. 44, 2322-2330. doi: 10.1249/MSS.0b013e3182667c2e

Vigelsø, A., Gram, M., Dybboe, R., Kuhlman, A. B., Prats, C., Greenhaff, P. L., et al. (2016). The effect of age and unilateral leg immobilization for 2 weeks on substrate utilization during moderate-intensity exercise in human skeletal muscle. J. Physiol. 594, 2339-2358. doi: 10.1113/JP271712 
Volpato, S., Bianchi, L., Lauretani, F., Lauretani, F., Bandinelli, S., Guralnik, J. M., et al. (2012). Role of muscle mass and muscle quality in the association between diabetes and gait speed. Diabetes Care 35, 1672-1679. doi: 10.2337/dc11-2202

Wackerhage, H., and Ratkevicius, A. (2008). Signal transduction pathways that regulate muscle growth. Essays Biochem. 44, 99-108. doi: 10.1042/BS E0440099

Wall, B. T., Dirks, M. L., Verdijk, L. B., Snijders, T., Hansen, D., Vranckx, P., et al. (2012). Neuromuscular electrical stimulation increases muscle protein synthesis in elderly type 2 diabetic men. Am. J. Physiol. 303, E614-E623. doi: 10.1152/ajpendo.00138.2012

Wall, B. T., Snijders, T., Senden, J. M., Ottenbros, C. L., Gijsen, A. P., Verdijk, L. B., et al. (2013). Disuse impairs the muscle protein synthetic response to protein ingestion in healthy men. J. Clin. Endocrinol. Metab. 98, 4872-4881. doi: $10.1210 /$ jc. $2013-2098$

West, D. W., Burd, N. A., Coffey, V. G., Baker, S. K., Burke, L. M., Hawley, J. A., et al. (2011). Rapid aminoacidemia enhances myofibrillar protein synthesis and anabolic intramuscular signaling responses after resistance exercise. Am. J. Clin.Nutr. 94, 795-803. doi: 10.3945/ajcn.111.013722

Wilkes, E. A., Selby, A. L., Atherton, P. J., Patel, R., Rankin, D., Smith, K., et al. (2009). Blunting of insulin inhibition of proteolysis in legs of older subjects may contribute to age-related sarcopenia. Am. J. Clin. Nutr. 90, 1343-1350. doi: 10.3945/ajcn.2009.27543

Wilkinson, S. B., Phillips, S. M., Atherton, P. J., Patel, R., Yarasheski, K. E., Tarnopolsky, M. A., et al. (2008). Differential effects of resistance and endurance exercise in the fed state on signalling molecule phosphorylation and protein synthesis in human muscle. J. Physiol. 586, 3701-3717. doi: 10.1113/jphysiol.2008.153916

Workeneh, B., and Bajaj, M. (2013). The regulation of muscle protein turnover in diabetes. Int. J. Biochem. Cell Biol. 45, 2239-2244. doi: 10.1016/j.biocel. 2013.06.028

Wu, C. L., Kandarian, S. C., and Jackman, R. W. (2011). Identification of genes that elicit disuse muscle atrophy via the transcription factors p50 and bcl-3. PloS ONE 6:e16171. doi: 10.1371/journal.pone.0016171

Zinna, E. M., and Yarasheski, K. E. (2003). Exercise treatment to counteract protein wasting of chronic diseases. Curr. Opin. Clin. Nutr. Metab. Care 6, 87-93. doi: 10.1097/00075197-200301000-00013

Zuo, L., and Pannell, B. K. (2015). Redox characterization of functioning skeletal muscle. Front. Physiol. 6:338. doi: 10.3389/fphys.2015.00338

Conflict of Interest Statement: The authors declare that the research was conducted in the absence of any commercial or financial relationships that could be construed as a potential conflict of interest.

Copyright (C) 2016 Rudrappa, Wilkinson, Greenhaff, Smith, Idris and Atherton. This is an open-access article distributed under the terms of the Creative Commons Attribution License (CC BY). The use, distribution or reproduction in other forums is permitted, provided the original author(s) or licensor are credited and that the original publication in this journal is cited, in accordance with accepted academic practice. No use, distribution or reproduction is permitted which does not comply with these terms. 